\title{
Single-Polymer Dynamics in Steady Shear Flow
}

\section{Douglas E. Smith, Hazen P. Babcock, Steven Chu*}

\begin{abstract}
The conformational dynamics of individual, flexible polymers in steady shear flow were directly observed by the use of video fluorescence microscopy. The probability distribution for the molecular extension was determined as a function of shear rate, $\dot{\gamma}$, for two different polymer relaxation times, $\tau$. In contrast to the behavior in pure elongational flow, the average polymer extension in shear flow does not display a sharp coil-stretch transition. Large, aperiodic temporal fluctuations were observed, consistent with end-over-end tumbling of the molecule. The rate of these fluctuations (relative to the relaxation rate) increased as the Weissenberg number, $\dot{\gamma} \tau$, was increased.
\end{abstract}

The dynamics of flexible polymers in shear is of great practical interest because this type of flow occurs whenever a fluid flows past a surface. Macroscopic, non-Newtonian rheological properties of polymer solutions, such as flow-dependent viscosities and normal stresses, result from microscopic stresses that arise when polymeric molecules are stretched and affect the solvent motion. Thus, much effort has been directed at predicting the molecular dynamics of polymers in shear flows (1-5). However, it has been difficult to rigorously test these predictions because the dynamics of a polymer molecule in shear have not been observed directly. Experimental efforts have mainly focused on measuring bulk rheological properties or on measuring the scattering of light or neutrons by polymer solutions $(6-10)$. Here we describe how single-molecule imaging techniques (11) can be used to study the configurations of polymers in shear flow so that the detailed molecular predictions of theories can be tested.

It has long been recognized that the amount of distortion of a molecule is strongly dependent on the nature of the flow $(4,5)$. In general, any planar flow of the form $\vec{v}=v_{x} \hat{x}+v_{y} \hat{y}$ may be represented as a linear superposition of a rotational flow with a vorticity $\omega=\left[\left(\partial v_{y} /\right.\right.$ $\left.\partial x)-\left(\partial v_{x} / \partial y\right)\right] / 2$ and an elongational flow with a strain rate $\dot{\varepsilon}=\left[\left(\partial v_{y} / \partial x\right)+\left(\partial v_{x} / \partial y\right)\right] / 2$. In a pure elongational flow $(\omega=0)$ one expects large deformations of a polymer, whereas in a pure rotational flow $(\dot{\varepsilon}=0)$ one expects only rotation and not deformation $(4,5)$.

Most practical flows consist of a mixture of both rotational and elongational components, and the resulting polymer deformation depends on the relative magnitudes of $\dot{\varepsilon}$ and $\omega$. In general, the response is not necessarily a linear superposition of the responses to each compo-

Departments of Physics and Applied Physics, Varian Building, Stanford University, Stanford, CA 94305, USA.

*To whom correspondence should be addressed. Email: schu@leland.stanford.edu nent. In a simple shear flow $\left(v_{y}=0\right.$ and $v_{x}=$ magnitude of the elongational and rotational components are equal ( $|\dot{\varepsilon}|=|\omega|)$ (Fig. 1A). In this case, it has been suggested that the polymer will not attain a stable, strongly stretched state $(4,5)$. In fact, large fluctuations in the extension due to an end-over-end tumbling of the molecule have been observed in some simulations (3). These fluctuations presumably occur because the stretched state is destabilized by the rotational component of the

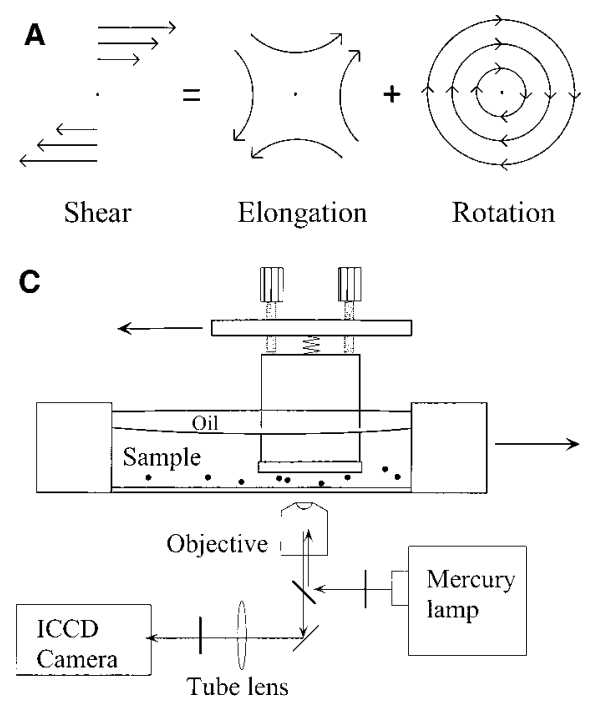
$\dot{\gamma} y$, where $\dot{\gamma}=d v_{x} / d y$ is the shear rate) the

shear flow (Fig. 1B). By observing individual molecules, we have experimentally confirmed the existence of such fluctuations and studied their statistical and temporal properties.

In our experiment, a uniform shear flow was created in a $\sim 50-\mu \mathrm{m}$ gap between two parallel glass plates (Fig. 1C) (12). Individual, isolated molecules of fluorescently labeled lambda bacteriophage DNA ( $\lambda$-DNA) in viscous sugar solutions were imaged at a depth of $25 \pm 15 \mu \mathrm{m}$ (13-15). These DNA molecules of contour length $L \cong 22 \mu \mathrm{m}$ may be considered "flexible" polymers because they contain roughly 440 persistence lengths (13). Images of the molecules were digitized, and a computer-generated cursor was used to measure (by inspection) the maximum extension, $x$, of the molecule along the shear direction.

The strength of an applied shear flow is specified by the dimensionless number $W i=$ $\dot{\gamma} \tau$ (called the Weissenberg number), which is the ratio of the applied shear rate, $\dot{\gamma}$, to the polymer's natural relaxation rate, $1 / \tau$. In our experiment, data were recorded at shear rates ranging from $\dot{\gamma}=0$ to $4.0 \mathrm{~s}^{-1}$ and with polymer relaxation times of $\tau=6.3$ and $19 \mathrm{~s}$ such that $W i$ ranged from 0 to 76 . The relaxation time was adjusted by using two different solvent viscosities, $\eta=60$ and 220 centipoise (cP) (16). Molecular extension data

B
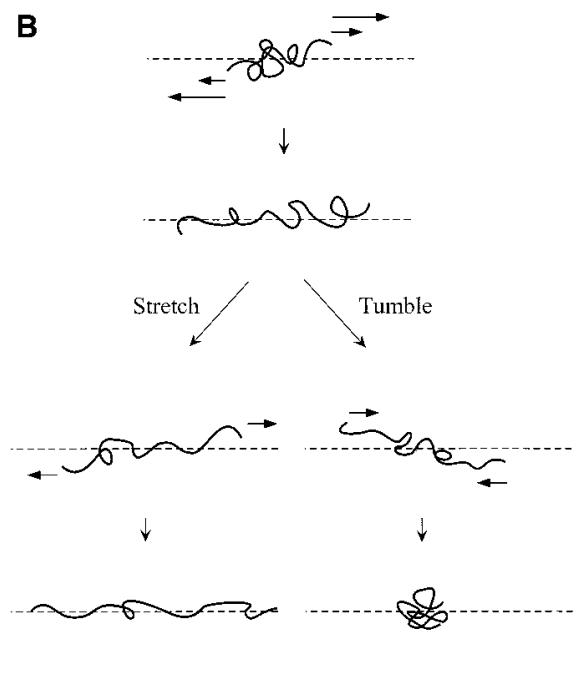

Fig. 1. (A) A simple shear flow may be represented as a superposition of a purely rotational flow and a purely elongational flow. (B) Schematic illustration of a flexible polymer stretching in a shear flow, as viewed in the polymer's center-of-mass frame. A velocity gradient perpendicular to the direction of flow gives rise to hydrodynamic drag forces across the molecule. These drag forces can overcome the entropic forces that tend to coil the chain and stretch the molecule along the shear axis (dashed line). As the polymer stretches, many different dynamical scenarios may occur. When the chain becomes aligned, the hydrodynamic drag decreases and the chain may begin to relax. However, Brownian motion can perturb a stretched chain so that it either continues to stretch or becomes unstable and tumbles end-over-end. (C) Schematic diagram of the apparatus. A uniform shear flow was created in a $\sim 50-\mu \mathrm{m}$ gap between two parallel plates by translating one glass plate parallel to another at constant speed. The top plate was a $1.3 \mathrm{~cm}$ by $2.5 \mathrm{~cm}$ glass slide and was leveled relative to the bottom plate with micrometer screws (12). Isolated molecules of fluorescently labeled $\lambda$-DNA (48,502 bp), represented here by dots, were imaged by fluorescence microscopy with an intensified video camera (ICCD). In order to follow a molecule in its center-of-mass frame, both plates were translated relative to the imaging optics in a direction opposite to the motion of the top shear plate. Mineral oil was placed on top of the aqueous sample to prevent evaporation. 


\section{REPOR T S}

(Fig. 2) were recorded after the mean extension reached a steady-state value. The initial transient period, during which the chain began to stretch, lasted for fewer than 50 units of strain $(\gamma=\dot{\gamma} t<50)$ for all of the recorded data sets. The raw data show that, even at relatively low $W i$, there are large fluctuations in the extension of the molecule. As $W i$ is increased, the molecule fluctuates more rapidly (relative to its relaxation rate) and reaches larger extensions. These two basic trends are in qualitative agreement with computer simulations (3). The amount of extension increases as $W i$ is increased because the velocity gradient, and hence the net hydrodynamic forces acting across the molecule, increase.

The exact configuration of the polymer backbone cannot be extracted from the images because of the limits of optical resolution $(\sim 0.3$ $\mu \mathrm{m})$. However, a reasonably detailed picture of the mass distribution (projected into a plane parallel to the shear plates) can be inferred from the relative brightness of various parts of the image (Fig. 3). After time-averaging many images, we find that, on average, the shape is approximately elliptical. However, the conformation changed continuously, and at various times dumbbell, half-dumbbell, kinked, and folded shapes, similar to those observed in elongational flow (11), were observed. Folded and kinked shapes were seen more often at higher $W i$. Lumps of mass density that propagated up or down the length of partially stretched chains were also seen (Fig. 3B). Molecules that devel-

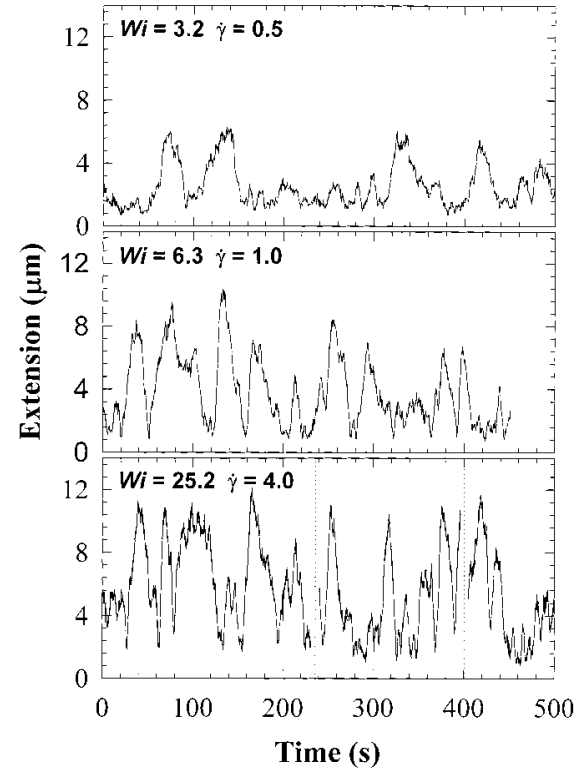

Fig. 2. Examples of typical extension versus time data for different Weissenberg numbers. Many data sets were recorded at each Wi with different molecules until a suitable amount of data for statistical analysis was obtained. The finite length of the shear channel limited the length of each data set. The vertical dotted lines in the $\mathrm{Wi}=$ 25.2 plot indicate where individual data sets begin and end. oped a hairpin fold (or U shape) while stretching or contracting tended to unfold slowly, like a rope traveling over a pulley (Fig. 3C). The polymer never made large excursions in a direction perpendicular to the shear plates. The depth of field of the microscope objective was about $1 \mu \mathrm{m}$, and we found that the entire chain contour could always be kept in focus as the polymer stretched.

To calculate the mean extension at each $W i$, we time-averaged the data over all of the measurements at each shear rate and viscosity (Fig. 4). In steady shear, the mean fractional extension, $\langle x\rangle / L$, increases gradually with $W i$ and appears to approach an asymptotic value of $\sim 0.4$ to 0.5 . This behavior differs markedly from that which occurs in pure elongational flow, where the extension rises very rapidly to a value close to the full contour length of the chain at relatively low strain rates (11). These steady-state results, in shear $(|\dot{\varepsilon}|=|\omega|)$ and in elongational flow $(\omega=0)$, qualitatively agree with the basic coil-stretch "phase diagram" picture proposed by de Gennes (5). However, whether or not the elongational component will dominate the behavior as soon as $|\dot{\varepsilon}|$ is made greater than $|\omega|$ remains to be explored (10). The sharp transition seen in elongational flow was not observed in birefringence measurements on DNA (17) because in the birefringence measurements there was a wide distribu-

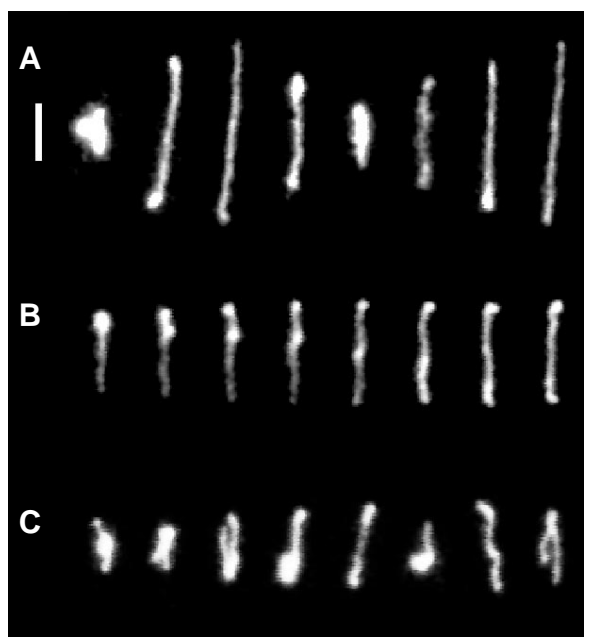

Fig. 3. Images of individual polymer chains undergoing conformational changes in steady shear with $W i=19$ and $\dot{\gamma}=1$. Each row of images is a series in time proceeding from left to right. The vertical bar at the left of the top row indicates $5 \mu \mathrm{m}$. (A) Example of a molecule that went from being coiled, to stretched, back to coiled, and then stretched again. The time interval between images is $6 \mathrm{~s}$. (B) Example of a lump of mass density that propagated down the contour of a partially stretched chain. The time interval between images is $0.84 \mathrm{~s}$. (C) Example of a molecule that became folded into an upward $U$ shape, then unraveled like a rope sliding over a pulley, then tumbled end-over-end and became folded in an inverted $U$ shape. The time interval between images is $6 \mathrm{~s}$. tion of different residence times of the molecules in the flow (18).

Previous experiments with light and neutron scattering indicated that polymers did not deform substantially in shear (7-9). However, this seems to be due to the small shear rates applied to the molecule in these experiments. Because of technical difficulties the scattering measurements were limited to $W i \lesssim 2.5$, where there is very little deformation. In our experiment we were able to reach a value of $W i=76$, and we saw substantial $(>100 \%)$ deformation for $W i \gtrsim$ 5 . The scattering experiments measure a different quantity than our experiment - they determine the components of the radius of gyration tensor of a time-averaged mass distribution. It is difficult to compare our small-deformation data with the scattering data because blooming of the image due to the intensified camera made it difficult to accurately estimate the radius of gyration of the coiled polymer when it is only slightly deformed. This is unfortunate because the values obtained in the light- and the neutron-scattering experiments do not agree. We do observe less deformation as $W i$ is increased than that predicted by the Zimm model (1). However, good agreement is not really expected, because the Zimm model is only meant to describe small fluctuations of the polymer about its equilibrium shape and does not constrain the polymer to have a finite extensibility.

Because we recorded the dynamics of individual molecules, the probability distribution for molecular extension in shear flow can be determined directly. Histograms of the extension were calculated by binning the extension data obtained from many molecules (Fig. 5). The extension is always positive because when the polymer is coiled we cannot usually distinguish the ends of the chain

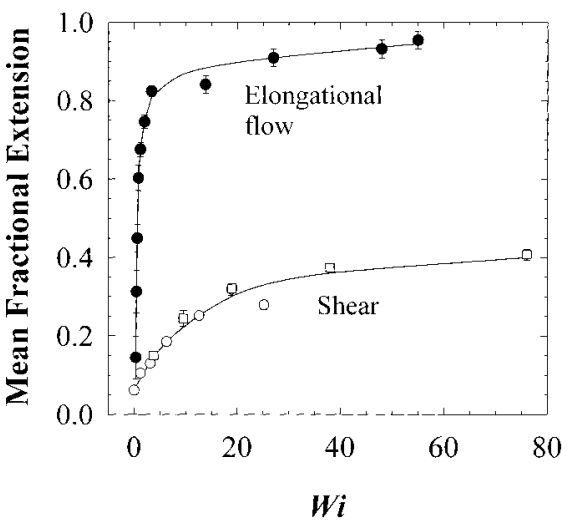

Fig. 4. The mean fractional extension was calculated by time averaging over each data set. The number of separate molecules on which the measurements for each data set were made varied from 6 to 40 , depending on $W i$. The results for both shear flow (open circles, $\eta=60 \mathrm{cP}$; open squares, $\eta=220 \mathrm{cP}$ ) and elongational flow (filled circles) are shown. Lines are to guide the eye. Error bars represent the standard error. 


\section{RE P O R T S}

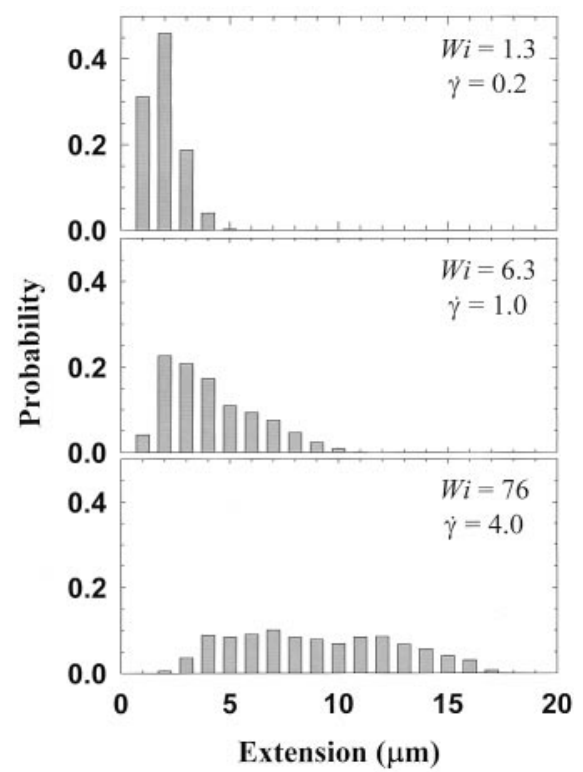

Fig. 5. Probability distributions for polymer extension in shear flow for several values of Wi. Histograms were calculated with $1-\mu \mathrm{m}$ bins and normalized by the total number of points ( $\sim 2000$ to 6000 points).

and therefore do not track the orientation of the end-to-end vector. The minimum measured extension is $\sim 1 \mu \mathrm{m}$, which is close to the inferred $\sim 0.7-\mu \mathrm{m}$ radius of gyration, $R_{\mathrm{G}, 0}$, of the coiled polymer at equilibrium (19). As $W i$ is increased, the shape of the extension distribution changes dramatically. At low values of $W i$, the distribution is strongly skewed to lower values, but as $W i$ is increased it becomes more symmetric: At higher shear rates, the molecules make more frequent and larger excursions in extension. The standard deviation, which indicates the size of the fluctuations, increases with $W i$ and reaches a value of $\sim 4 \mu \mathrm{m}(\sim 20 \%$ of the full contour length) for $W i=76$.

To investigate the spectral properties of the fluctuations, we calculated the power spectral density per unit time (PSD) using a fast Fourier transform (Fig. 6A) (20). The rotational component of a simple shear flow causes solid objects of finite aspect ratio, such as ellipsoids (21), to tumble periodically in steady shear flow and might lead one to expect periodic tumbling for a polymer coil (2). However, these simplest models neglect Brownian motion. When Brownian motion is important we might expect a tumbling motion that is stochastic rather than periodic. Indeed, no distinct peaks were distinguishable in the power spectra, indicating that there was no periodicity detectable within the observed frequency range (22). The data suggest a random stretching and contraction process that occurs more frequently at higher shear rates. As $\dot{\gamma}$ is increased, the fluctuations are larger and more frequent because both the stretching and tumbling are driven by the velocity gradient as indicated in Fig. 1B. Alterna-

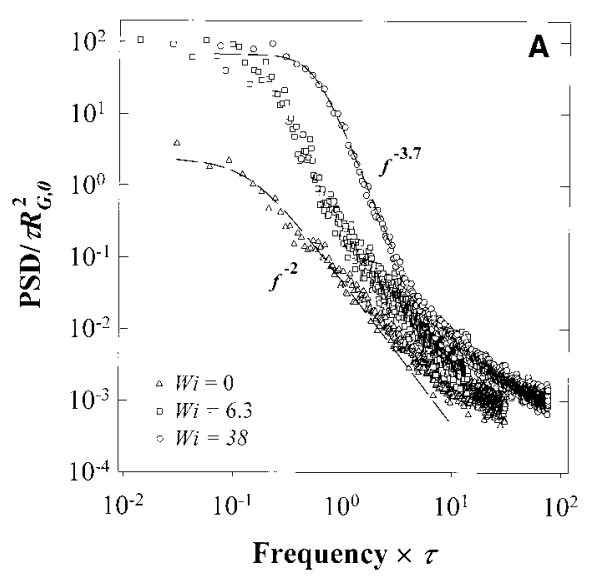

Fig. 6. (A) Plots of the power spectral density per unit time (PSD) of the extension fluctuations for various values of Wi. Here we have expressed the PSD in dimensionless units on the basis of known values of $R_{\mathrm{G}, \mathrm{O}}$ and $\tau$. At equilibrium (Wi $=0)$ the shape of the PSD is close to that of a Lorentzian function (dashed line), PSD $\sim 1 /\left(a+f^{2}\right)$, where $a$ is a constant. When the shear flow is applied the PSD increasingly deviates from this form; it rolls off faster at higher frequencies. The other dashed line shows the behavior of a function proportional to $1 /\left(a+f^{3.7}\right)$. (B) Dimensionless PSD plot for two data sets with different shear rates and relaxation times (different solvent viscosities) but the same value of Wi. The data sets overlap as expected theoretically. (C) The autocorrelation function $\langle x(t) x(t+T)\rangle$ plotted versus time delay, $T$, for various Wi. $\langle x(t) x(t+T)\rangle$ was calculated from each data set after subtracting the mean extension value. The products of each pair of data points were collected in 1-s time interval bins by rounding the time difference between each pair of points to the nearest second. The results are normalized so that $\langle x(t) x(t)\rangle=1.0$.

the validity of this assumption, we plotted $\mathrm{PSD} / \tau R_{\mathrm{G}, 0}{ }^{2}$ (dimensionless PSD) versus frequency $\times \tau$ (dimensionless frequency) for two data sets that had the same value of $W i$ but were acquired at different viscosities and shear rates (Fig. 6B). In agreement with theoretical expectations, we found that the PSD for these data sets do collapse when plotted with these dimensionless parameters.

In zero flow $(W i=0)$, there are still fluctuations in the extension that are entirely due to Brownian motion. We find that at low and intermediate frequencies the PSD of these small fluctuations from equilibrium is reasonably well fit by a Lorentzian function, PSD $\sim 1 /(a+$ $f^{2}$ ), where $a$ is a constant. This function is the form of the PSD for a Brownian particle confined by a harmonic potential well (24), which indicates that in this frequency range the dynamics may be described by a "dumbbell" model in which the polymer is modeled as two Brownian particles connected by a harmonic spring. When a shear flow is applied, the shape of the PSD function changes. For the lowest $W i$ studied ( $W i=1.3, \dot{\gamma}=0.2$ ), the chain is only weakly deformed and the PSD deviates only slightly from the Lorentzian form. As $W i$ is increased, the deviation increases because the PSD falls off faster at intermediate frequencies. In all cases, however, the PSD declined much more slowly than the Lorentzian at the highest observed frequencies. This behavior is also seen in some computer simulations of polymers in shear (25).

Another way of characterizing the temporal properties of random fluctuations is to calculate the autocorrelation function $\langle x(t) x(t$ $+T)\rangle$, where $t$ is time and $T$ is a fixed time delay interval. The autocorrelation decay shows that, although the fluctuations are not periodic, they occur on a characteristic time 


\title{
R E P O R T S
}

scale that depends on the shear rate. The autocorrelation function may also be plotted against a dimensionless time, namely the amount of strain $\dot{\gamma} T$ (Fig. 6C). When plotted this way, one sees that the deformation remains correlated for higher strains at higher $W i$. Larger changes in conformation occur at higher $W i$, and more strain is apparently required to produce these changes. Empirically, the data for all $W i$ may be approximately, but not exactly, collapsed by plotting the autocorrelation versus $\dot{\gamma} T /(W i)^{2 / 3}$.

We have presented experimental data on the conformational dynamics of individual polymer molecules in steady shear flow. Possible improvements in the technique include better spatial and temporal resolution and specific labeling of portions of the polymer. The ability to clearly distinguish the ends of the chain should allow the tumbling dynamics to be studied in greater detail. In future studies, it is expected that these types of measurements may be extended to time-dependent (transient) flows and to the study of semi-dilute and entangled polymer solutions. Such experiments, in conjunction with the development of accurate molecular models, should reveal many details of the molecular processes that underlie non-Newtonian rheological effects. This approach should be useful for rigorously testing polymer physics models and lay the foundation for a complete microscopic understanding of the rheology of polymer solutions.

References and Notes

1. P. Rouse, J. Chem. Phys. 21, 1272 (1953); B. Zimm ibid. 24, 269 (1956); A. Peterlin, W. Heller, M. Nakagaki, ibid. 28, 470 (1958); H. Warner, Ind. Eng. Chem. Fundam. 11, 379 (1972); E. Hinch, J. Fluid Mech. 75, 765 (1976); J. Magda, R. Larson, M. Mackay, J. Chem. Phys. 89, 2504 (1988); L. Wedgewood and H. Ottinger, J. Non-Newtonian Fluid Mech. 27, 245 (1988); L. Wedgewood, D. Ostrov, R. Bird, ibid. 40, 119 (1991).

2. W. Kuhn and H. Kuhn, Helv. Chim. Acta. 26, 1394 (1943); D. E. Keyes and F. H. Abernathy, J. Fluid Mech. 185, 503 (1987)

3. T. Liu, J. Chem. Phys. 90, 5826 (1989); P. Doyle, E. Shaqfeh, A. Gast, J. Fluid Mech. 334, 251 (1997); P. Doyle, E. Shaqfeh, J. Non-Newtonian Fluid Mech. 76 78 (1998)

4. J. Lumley, Annu. Rev. Fluid Mech. 1, 367 (1969).

5. P. G. de Gennes, J. Chem. Phys. 60, 5030 (1974)

6. R. Larson, Constitutive Equations for Polymer Melts and Solutions (Buttersworths, New York, 1988).

7. F. Cottrell, E. Merrill, K. Smith, J. Polym. Sci. Polym. Phys. Ed. 7, 1415 (1969); A. Link and J. Springer. Macromolecules 26, 464 (1993).

8. E. C. Lee, M. J. Solomon, S. J. Muller, Macromolecules 30, 7313 (1997)

9. P. Lindner and R. Oberthur, Colliod Polym. Sci. 266, 886 (1988)

10. G. G. Fuller and L. G. Leal, Rheol. Acta 19, 580 (1980); J. Bossart and H. Ottinger, Macromolecules 30, 5527 (1997).

11. D. E. Smith and S. Chu, Science 281, 1335 (1998); T. T. Perkins, D. E. Smith, S. Chu, ibid. 276, 2016 (1997); __ in Flexible Chain Dynamics in Elongational Flow, H. Kausch and T. Nguyen, Eds. (Springer-Verlag, Berlin, in press).

12. A $1.3 \mathrm{~cm}$ by $2.5 \mathrm{~cm}$ glass slide glued to a piece of Plexiglass (acrylic plastic) was positioned above a 2.5 $\mathrm{cm}$ by $5.7 \mathrm{~cm}$ by $1.5 \mathrm{~cm}$ channel by three micrometer screws. The plate was held against the screws by a stiff spring, allowing one to level the plates and to adjust the size of the gap between them (with $<5 \%$ variation). The bottom plate, which acted as an optical window, was a $1.5 \mathrm{~cm}$ by $5.7 \mathrm{~cm}$, no. 2 glass coverslip. The top plate was connected to the channel by a translation stage that was leveled relative to the bottom plate by a fourth micrometer screw. The translation stage was driven by an optically encoded dc motor moving at speeds of 10 to $200 \mu \mathrm{m} / \mathrm{s}$ with $2 \%$ root mean square variation over $2.4 \mathrm{~cm}$ of travel.

13. $\lambda$-DNA (Gibco BRL, Gaithersburg, MD) was labeled with YOYO-1 (Molecular Probes, Eugene, OR) at a dye/base pair ratio of 1:4 for $>1$ hour. The persistence length of native DNA is $\sim 53 \mathrm{~nm}$ [C. Bustamante, J. Marko, E. Siggia, S. Smith, Science 265, 1599 (1994)] and its hydrodynamic diameter is $\sim 2 \mathrm{~nm}$ [R Pecora, ibid. 251, 893 (1991)]. When labeled with YOYO, the contour length increases to $\sim 22 \mu \mathrm{m}[T$. T. Perkins, D. E. Smith, R. G. Larson, S. Chu, ibid. 268, 83 (1995)]. Experiments were performed at $\sim 20^{\circ} \mathrm{C}$ in a $\mathrm{pH} 8$ buffer consisting of $10 \mathrm{mM}$ tris- $\mathrm{HCl}, 2 \mathrm{mM}$ EDTA, $10 \mathrm{mM} \mathrm{NaCl}, 4 \%$-mercaptoethanol, glucose oxidase $(\sim 50 \mu \mathrm{g} / \mathrm{ml})$ and catalase $(\sim 10 \mu \mathrm{g} / \mathrm{ml}), \sim 10$ to $18 \%(\mathrm{w} / \mathrm{w})$ glucose, and 40 to $55 \%(\mathrm{w} / \mathrm{w})$ sucrose. In this solution, where the free oxygen has been minimized, the photobleaching of the molecules during the measurements was found to be negligible. The viscosity of each solution was measured and adjusted by varying the sugar concentrations.

14. Molecules were epi-illuminated by a $100 \mathrm{~W}$ mercury arc lamp (Zeiss) with a $470 \pm 32 \mathrm{~nm}$ bandpass excitation filter and a 500-nm long-pass dichroic mirror and imaged with a $\times 60,1.2$ numerical aperture water immersion objective (Nikon), a 160-mmto-infinity-corrected conversion lens (Zeiss), a 40$\mathrm{cm}$ tube lens, a 515-nm long-pass emission filter, a microchannel plate intensifier (Hamamatsu), and a video camera (Phillips CCD).

15. The concentration was kept very low so that the dynamics of isolated, noninteracting molecules could be observed. Typically it was $10^{4}$ to $10^{5}$ times lower than the concentration $\left(c^{*}\right)$ at which coiled molecules begin to overlap.
16. We determined that $\tau=6.3 \mathrm{~s}$ in the 60 -cP solution and $19 \mathrm{~s}$ in the $220-\mathrm{CP}$ solution by analyzing the relaxation of $>40$ molecules that were extended by $>30 \%$ when the flow was stopped. $\tau$ was determined by fitting to the function $x(t)^{2}=c \exp (-t / \tau)+2 R_{\mathrm{G}^{\prime}}$ where $\tau, c$, and $R_{\mathrm{G}}$ were free parameters.

17. E. Atkins and M. Taylor, Biopolymers 32, 911 (1992).

18. Also, the birefringence does not directly correspond to the extension but rather the ordering of portions of the chain along the polarization axis.

19. D. E. Smith, T. T. Perkins, S. Chu, Macromolecules 29 1372 (1996)

20. The FFT was calculated after subtracting the mean extension from all of the data points and multiplying the data by a Welch window function [W. H. Press et al., Numerical Recipes in C (Cambridge Univ. Press, Cambridge, 1988), p. 442]. The PSD for all of the data sets at the same $\dot{\gamma}$ and $\eta$ were averaged together and normalized according to Parseval's theorem.

21. G. Jeffery, Proc. R. Soc. Lond. Ser. A 102, 161 (1922).

22. The maximum frequency that can be resolved is determined by the sampling rate, typically $0.1 \mathrm{~s}$. The minimum frequency is determined by the length of the run, typically $150 \mathrm{~s}$ for $\dot{\gamma}>2$ and as long as $800 \mathrm{~s}$ for $\dot{\gamma}=0.2$.

23. The ratio of the values determined experimentally (16) were close to, but not exactly equal to, the ratio of the solvent viscosities. The deviation from a strict linear proportionality may be due to slight differences in the solvent arising from the differing fractions of water in the two solutions.

24. M. Wang and G. Uhlenbeck, Rev. Mod. Phys. 17, 323 (1945).

25. E. Shaqfeh and J. Hur, personal communication.

26. We acknowledge assistance from T. Perkins with an earlier version of this experiment and helpful comments from J. Hur, R. Larson, T. Perkins, E. Shaqfeh, and B. Zimm. This work was supported in part by the AFOSR and the NSF. D.E.S received support from a fellowship from the NSF Program in Mathematics and Molecular Biology. H.P.B. was supported in part by an $\mathrm{NIH}$ biophysics training grant.

3 December 1998; accepted 11 February 1999

\section{How Strong Is a Covalent Bond?}

\author{
Michel Grandbois, ${ }^{1}$ Martin Beyer, ${ }^{2}$ Matthias Rief, ${ }^{1,3}$ \\ Hauke Clausen-Schaumann, ${ }^{1}$ Hermann E. Gaub ${ }^{1 *}$
}

\begin{abstract}
The rupture force of single covalent bonds under an external load was measured with an atomic force microscope (AFM). Single polysaccharide molecules were covalently anchored between a surface and an AFM tip and then stretched until they became detached. By using different surface chemistries for the attachment, it was found that the silicon-carbon bond ruptured at $2.0 \pm 0.3$ nanonewtons, whereas the sulfur-gold anchor ruptured at $1.4 \pm 0.3$ nanonewtons at force-loading rates of 10 nanonewtons per second. Bond rupture probability calculations that were based on density functional theory corroborate the measured values.
\end{abstract}

The mechanical stability of covalent bonds has been investigated indirectly in ensemble measurements or by flow-induced chain frac-

${ }^{1}$ Lehrstuhl für Angewandte Physik, Ludwig-Maximilians-Universität, Amalienstrasse 54, D-80799 München, Germany. ${ }^{2}$ Institut für Physikalische und Theoretische Chemie, Technische Universität München, Lichtenbergstrasse 4, 85748 Garching, Germany. ${ }^{3}$ Department of Biochemistry B400, School of Medicine, Stanford University, Stanford, CA 943055307, USA.

*To whom correspondence should be addressed. ture in liquids (1). The development of nanoscale manipulation techniques (2) has made it possible to directly address single atoms or molecules and probe their mechanical properties. It has been shown that individual polymers may be stretched between the tip of an AFM cantilever and a substrate surface (3-12). In these studies, polymers were coupled either by specific receptor ligand systems, which were covalently attached to the polymers $(8)$, or by nonspecific adsorption to the tip and the substrate (3, 